\title{
Spatiotemporal interplay of severe acute respiratory syndrome coronavirus and respiratory mucosal cells drives viral dissemination in rhesus macaques
}

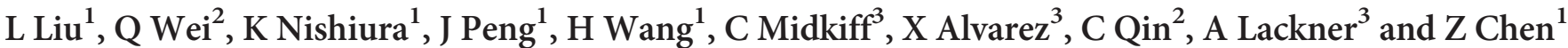

Innate immune responses have a critical role in the control of early virus replication and dissemination. It remains unknown, however, how severe acute respiratory syndrome coronavirus (SARS-CoV) evades respiratory innate immunity to establish a systemic infection. Here we show in Chinese macaques that SARS-CoV traversed the mucosa through the respiratory tract within 2 days, resulting in extensive mucosal infiltration by Tcells, MAC387 ${ }^{+}$, and $\mathrm{CD}_{163}{ }^{+}$ monocytes/macrophages followed by limited viral replication in the lung but persistent viral shedding into the upper airway. Mucosal monocytes/macrophages sequestered virions in intracellular vesicles together with infected Langerhans cells and migrated into the tonsils and/or draining lymph nodes within 2 days. In lymphoid tissues, viral RNA and proteins were detected in infected monocytes upon differentiation into dendritic cells (DCs) within 3 days. Systemic viral dissemination was observed within 7 days. This study provides a comprehensive overview of the spatiotemporal interactions of SARS-CoV, monocytes/macrophages, and the DC network in mucosal tissues and highlights the fact that, while these innate cells contribute to viral clearance, they probably also serve as shelters and vehicles to provide a mechanism for the virus to escape host mucosal innate immunity and disseminate systemically.

\section{INTRODUCTION}

Severe acute respiratory syndrome (SARS) is a highly contagious and life-threatening human respiratory disease caused by a zoonotic coronavirus (SARS-CoV). ${ }^{1}$ The global transmission of SARS-CoV is dependent on the capacity of this virus to efficiently cross from one host to the next by traversing the respiratory mucosa and overcoming host defenses to establish an infection, but the underlying mechanisms remain unclear. It is surprising that our knowledge of the immune events in the upper respiratory mucosa, in particular during acute respiratory infection, remains rather limited. Although the SARS epidemic was successfully contained by July 2003, there is serious concern regarding a recurrence of severe $\mathrm{CoV}$-mediated disease. In particular, the Middle East Respiratory Syndrome coronavirus has recently been identified as the etiological agent of a severe respiratory illness in patients from Saudi Arabia, Qatar, Jordan, and the United Kingdom. ${ }^{2,3}$ Unraveling how upper airway mucosal exposure of SARS-CoV results in the establishment of infection and subsequent systemic virus dissemination is critical to understand its pathogenesis and to identify key steps for the future prevention of related disease.

The respiratory mucosa constitutes not only a natural barrier to pathogen invasion but also serves as an important gateway for respiratory infection. Many viruses, including SARS-CoV, breach this mucosal barrier by infecting the epithelium directly. ${ }^{4,5}$ After an initial amplification at the entry site, viruses interact with various immune cells, including dendritic cells (DCs), macrophages, and T cells, beneath the epithelium. ${ }^{6}$ Previous studies showed that these immune cells differ in their ability to induce an innate immune response against virus and

${ }^{1}$ AIDS Institute and Research Center for Infection and Immunology, Department of Microbiology, Li Ka Shing Faculty of Medicine, The University of Hong Kong, Hong Kong SAR, P.R. China. ${ }^{2}$ Institute of Laboratory Animal Science, Chinese Academy of Medical Sciences (CAMS) and Peking Union Medical College (PUMC), Beijing, P.R. China and ${ }^{3}$ Division of Comparative Pathology, Tulane National Primate Research Center, Covington, Louisiana, USA. Correspondence: L Liu or Z Chen (liuli71@hku.hk or zchenai@hku.hk) 
in their roles in either the elimination or dissemination of viral infection. The roles they have greatly depend on their activation or differentiation status, as well as their susceptibility to virus infection and replication. ${ }^{7-9}$ For example, during early lymphocytic choriomeningitis virus infection, the virus targets monocytes/macrophages and DCs, contributing to immunosuppression that leads to persistent infection. ${ }^{7,8}$ During vaccinia virus infection, however, despite the fact that vaccinia virus also infects inflammatory monocytes, $\mathrm{CD}^{+} \mathrm{T}$ cells pursue and lyse infected inflammatory monocytes and eliminate the infection at the primary site. ${ }^{10}$ In the case of HIV-1 infection, it is the activated mucosal $\mathrm{CD} 38^{+} \mathrm{CD} 4{ }^{+} \mathrm{T}$ cells that are crucial for infection and dissemination of HIV-1 through the mucosa of the female lower genital tract. ${ }^{11}$

Type I alveolar epithelium is the predominant target for SARS-CoV infection, and macrophages were found to be subsequently infected in patients who died of SARS. ${ }^{4}$ Infection leads to intense innate immune responses, which contribute to lung pathology. Patients also have evidence of systemic infection as indicated by other organ and immune dysfunctions, including prominent gastrointestinal symptoms of diarrhea, splenic atrophy, and lymphadenopathy. ${ }^{12-14}$ Among these, acute renal failure in SARS patients usually has a catastrophic outcome, with a mortality rate of $77 \% .{ }^{15}$ Consistently, previous reports have described SARS viral particles and genomic sequences in urine, the mucosa of the intestine, the epithelium of the renal distal tubules, the neurons of the brain, circulating lymphocytes, and lymphoid tissues. $4,16,17$ Although much is known about the terminal phase of SARS, little is known about the early events during the acute phase of infection. The early cellular target(s) of infection remain elusive, as do the innate immune response during acute infection, particularly in the upper respiratory tract, which is the initial site for SARS-CoV infection and replication, and the roles of innate cells in disease progression. Understanding of these factors requires robust knowledge of the phenotypes and functional features of the cells in the respiratory mucosa that are implicated in the early events of SARS-CoV infection, which is difficult to study in SARS-CoV-infected humans owing to difficulties in collecting samples during the acute phase of infection.

We have recently demonstrated that the primary receptor of SARS-CoV, angiotensin-converting enzyme 2 (ACE2), is widely distributed in the upper respiratory tract and the lungs of Chinese rhesus macaques and supports viral entry as efficiently as its human homolog. ${ }^{18-20}$ Moreover, epithelial cells lining the salivary gland duct and alveolar pneumocytes are early cellular targets in Chinese rhesus macaques that are experimentally infected with pathogenic SARS-CoV. ${ }^{20}$ In this study, we further investigated the kinetics of virus replication, cellular targets, and the innate immune response during acute phase of SARS-CoV infection in Chinese rhesus macaques. Based on these studies, we present a previously unrecognized model demonstrating the mechanism underlying the mucosal innate activation of macrophages, as well as the promotion of mucosal transmission and dissemination of SARS-CoV by a DC subpopulation.

\section{RESULTS}

Predominant epithelial cellular targets and differential kinetics of SARS-CoV replication in the upper and lower respiratory mucosa

In this study, 12 rhesus macaques (Macaca mulatta) of Chinese origin were challenged intranasally with the live pathogenic SARS-CoV PUMC $\left(10^{5}\right.$ TCID $\left._{50}\right)$ as previously described. ${ }^{20}$ Four infected macaques were killed at 2, 3, and 7 days postinoculation (dpi). An additional three mock-inoculated animals served as negative controls (Figure 1a). Although there were no remarkable changes in leukocyte counts and clinical signs such as cough, upper respiratory tract catarrh, dyspnea, loss of appetite, vomiting, rash, and diarrhea were absent, all infected animals did develop fever $\left(\sim 40{ }^{\circ} \mathrm{C}\right)$. SARS-CoV RNA was readily detected by real-time reverse transcriptase-PCR (RT-PCR) in oral swabs from all infected macaques at multiple time points tested (Figure 1b), peaking at 1 or $2 \mathrm{dpi}$, with a slight decrease over the following days. By day 7 , viral loads were maintained at similar levels in three out of four macaques, AD0516, AD0517, and AD0518, but were undetectable in one macaque (AD0515) (Figure 1b). In contrast, SARS-CoV RNA was detected in lung homogenates from two out of four macaques at $2 \mathrm{dpi}$ (AD0506 and AD0508) and one of four macaques at 3 and 7 dpi only (AD0513 and AD0516), suggesting a trend towards decreased viral replication over time in the lungs. Hematoxylin and eosin staining of lung tissues collected at the time of necropsy (2-7 dpi) revealed mild damage as indicated by thickened alveolar septa without the typical diffuse alveolar damage found among SARS patients at the time of necropsy (2-7 dpi) (Figure 1c).

Consistent with our previous findings, ${ }^{20}$ positive immunohistochemical (IHC) signals for the viral nucleoprotein (NP) were found in the epithelia of the salivary gland ducts and the trachea, as well as alveolar pneumocytes at 2 dpi (Figure 2). Further extensive IHC analysis revealed $\mathrm{NP}^{+}$epithelial cells along the entire respiratory tract within $2 \mathrm{dpi}$ in infected animals but not in control animals. In agreement with viral RNA detection by RT-PCR (Figure 1b), NP signals were predominant in the pharynx mucosa and the lungs by day 2 (Table 2 and Figure 2). Subsequently, more NP signals were detected at $7 \mathrm{dpi}$ in the epithelium lining the nasal cavity and the vascular endothelium beneath the pharyngeal mucosa (Figure 2a,e), suggesting a time lag for viral dissemination. In the lungs, $\mathrm{NP}^{+}$alveolar pneumocytes were primarily found at 2 dpi (2/4 monkeys, AD0506 and AD0508) and infrequently at 3 and 7 dpi (1/8 monkeys, AD0518) (Table 1). Further analysis via detection of epithelial membrane antigen (AE1/AE3), viral $\mathrm{N}$ protein, and viral genome RNA, however, demonstrated concentrated signals for both NP and viral RNA in AE1/AE3 ${ }^{+}$ cells in the lungs at 2 dpi (2/4 monkeys, AD0506 and AD0508) and 7 dpi (AD0518), but only NP signals were detected in the upper respiratory mucosa, indicating a higher efficiency of viral replication in alveolar pneumocytes than in the upper respiratory mucosal epithelium during acute infection (Figure 3 and Table 1). Collectively, these findings demonstrated that a broad range of mucosal epithelial cells could be targeted for 
a

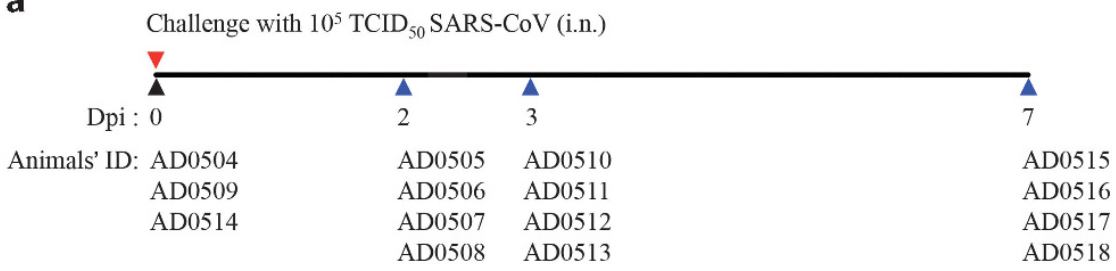

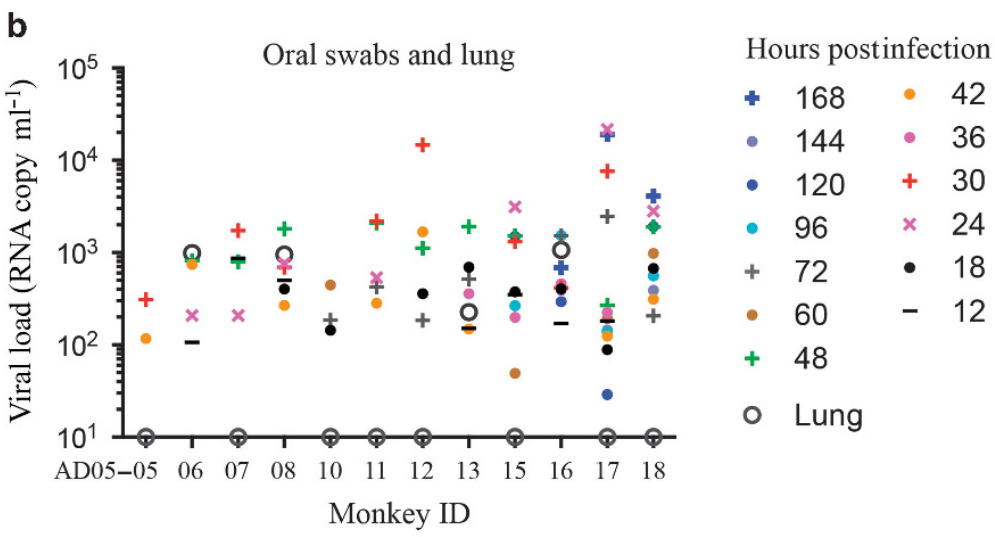

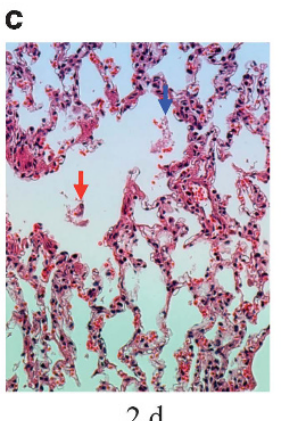

$2 \mathrm{~d}$

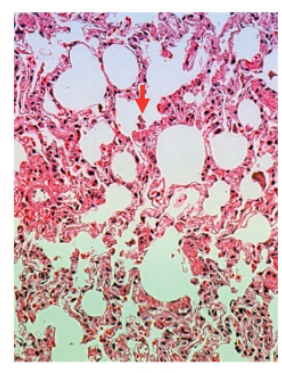

$3 \mathrm{~d}$

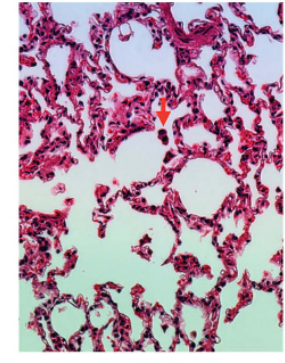

$7 \mathrm{~d}$

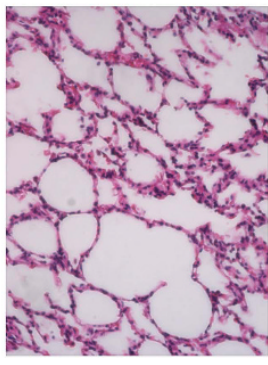

Neg

Figure 1 Intranasal inoculation of Chinese rhesus macaques with severe acute respiratory syndrome coronavirus (SARS-CoV). (a) Experimental scheme for virus challenge. A total of 12 Chinese rhesus macaques were challenged with live pathogenic SARS-CoV $\left(10^{5} \mathrm{TCID}_{50}\right)$ via the nasal cavity. Four animals each were killed at 2, 3, and 7 days postinoculation (dpi). Three additional uninfected macaques were challenged with phosphate-buffered saline as negative controls. (b) Detection of viral RNA in oral swabs and lungs. SARS-CoV RNA was detected by nested reverse transcriptase-PCR in the swabs at multiple time points postinfection from all 12 monkeys and in lung homogenates from two out of four macaques at 2 dpi (AD0506 and AD0508) and one out of four macaques at 3 and $7 \mathrm{dpi}$ (AD0513 and AD0516). (c) Examples of hematoxylin and eosin staining of lung tissue. Macaques inoculated with SARS-CoV exhibited slightly thickened alveolar septa and some monocyte infiltration at days 2, 3, and 7 (red arrows) with epithelial cell debris present in the alveolar lumen at day 2 (2 days, blue arrows). Macaques inoculated with cell culture medium exhibited normal alveolar septa, intact reticular fibers, and epithelial lining (Neg). Each image is representative of at least three monkeys.

SARS-CoV seeding in the respiratory mucosal tissues, which is in line with the distribution of ACE2 ${ }^{+}$cells as we previously described. ${ }^{20}$ Moreover, the results suggest a trend toward rapid control of epithelial infection in the lungs and more persistent viral production in the upper airway mucosa.

\section{Viral targeting of inflammatory monocytes/macrophages and Langerhans cells in the upper airway mucosa}

The lamina propria is rich in immune cells, includ ing macrophages, DCs, and lymphoid cells. Moreover, infected epithelium also induces the migration of circulating blood monocytes, macrophages, and lymphocytes to the inflamed site of infection, rendering it a key place for immune responses to occur. ${ }^{6}$ Beneath respiratory epithelial cells, $\mathrm{NP}^{+}$immune cells were readily detected within the lamina propria of the pharyngeal mucosa of all infected animals (Figure $\mathbf{2 b}-\mathbf{e}$ ). To understand the innate immune response and define the phenotypes of these $\mathrm{NP}^{+}$cells, IHC double staining with a SARS NP antibody and antibodies for lymphocytes (CD3, CD20), macrophages (MAC387, CD68, CD163), or DCs (DC-specific intercellular adhesion molecule3-grabbing non-integrin (DC-SIGN), Langerin) was performed. In infected animals but not in control animals, the pharyngeal mucosa exhibited strong staining of monocytes/ macrophages, indicating monocyte/macrophage infiltra tion as early as 2 dpi (Figure 4 and see Supplementary Figure S1 online). These monocytes/macrophages include recently infiltrated monocytes/macrophages $\left(\mathrm{MAC}_{3} 87^{+}\right)$, as well as activated/inflammatory macrophages $\left(\mathrm{CD} 163^{+}\right)$ (see Supplementary Figure S1). In contrast, $\mathrm{CD}^{+} 8^{+}$ 


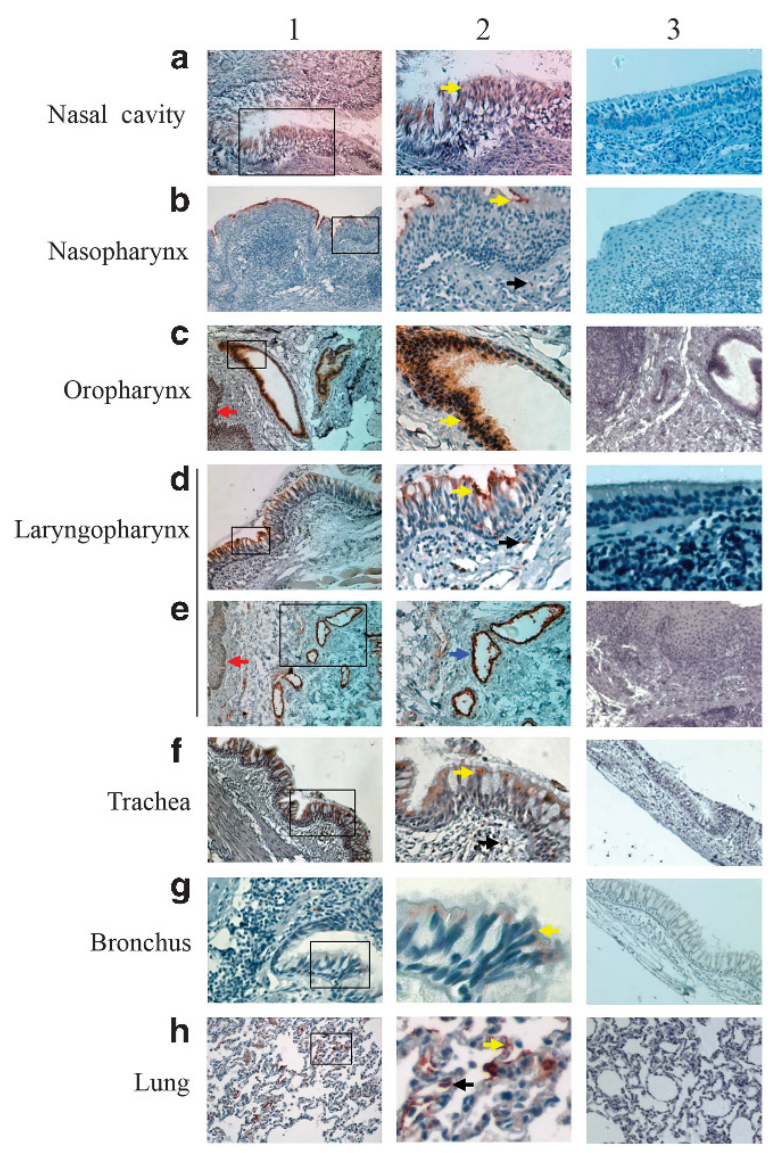

Figure 2 Localization of severe acute respiratory syndrome coronavirus (SARS-CoV) infection in the mucosa of the respiratory tract. Examples of tissue sections stained with anti-nucleoprotein (red) and hematoxylin (blue). Panel 1 shows a low magnification overview $(\times 200)$. Panel 2 shows a higher magnification of the boxed area in panel $1(x 400)$. Panel 3 shows the same tissue from an uninfected monkey $(\times 200)$. (a) In the nasal cavity, $\mathrm{NP}^{+}$cells were identified in the epithelial lining at 7 days postinoculation (dpi) (a2, yellow arrow). In the (b and c) pharynx and (d and e) laryngopharynx, $\mathrm{NP}^{+}$cells were identified at the epithelium of salivary gland ducts at 2 and 3 dpi (c2, yellow arrow), the basal layer of the epidermis (c1 and $\mathbf{e} 1$, red arrow), the lamina propria (b2 and $\mathbf{d} 2$, black arrow), and the epithelial lining of the mucosa (b2 and d2, yellow arrow) at 2,3 , and $7 \mathrm{dpi}$ and the vascular endothelium (e2, blue arrow) at $7 \mathrm{dpi}$. (f) In the trachea, $\mathrm{NP}^{+}$cells were found in the epithelial lining (f2, yellow arrow) and lamina propria (f2, black arrow). (g) In the bronchus, NP ${ }^{+}$cells were identified in the epithelial lining (g2, yellow arrow). (h) In the lungs, $\mathrm{NP}^{+}$ were observed on type I pneumocytes (h2, yellow arrow) and infiltrated immunocytes (h2, black arrow).

monocytes/macrophages and Langerin ${ }^{+}$cells (LCs) were identified in the lamina propria in both infected and control animals (see Supplementary Figure S1). Interestingly, whereas the majority of $\mathrm{CD}_{163}{ }^{+}$and nearly all $\mathrm{CD}^{+} 8^{+}$macrophages were localized beneath the epithelium in the lamina propria or mucosa-associated lymph tissue (MALT) (Figure 4a,b and see Supplementary Figure S1), many $\mathrm{MAC}_{387^{+}}$and some $\mathrm{CD} 163^{+}$monocytes/macrophages were found within and surrounding the epithelial layers targeted by SARS-CoV (Figure $\mathbf{4 b}$ and see Supplementary Figure S1), suggesting a possible role for these cells in the control of epithelial infection.
In the pharynx, NP signals were detected in the intracellular compartment containing $\mathrm{CD} 63^{+}$monocytes/macrophages within the epithelium, the intracellular compartment, or cytoplasm of $\mathrm{CD}^{+} 8^{+}$monocytes/macrophages beneath the epithelium and the cytoplasm of DCs (Langerin ${ }^{+}$) (Figure 4) but not in lymphocytes (CD3 and CD20) and MAC387 ${ }^{+}$ monocytes/macrophages (Table 2). The positive staining for $\mathrm{NP}$ in $\mathrm{CD} 163^{+}$and $\mathrm{CD} 68^{+}$monocytes/macrophages and LCs suggests that the virus infected these cells within the pharyngeal mucosa. Indeed, double staining with antibodies for the SARS-CoV receptor ACE2 and cell markers showed that, in addition to alveolar pneumocytes, bronchial epithelium, and vascular endothelial cells, many immune cells demonstrated even stronger positive signals for ACE2 in the lungs (see Supplementary Figure S2A). These cells include $\mathrm{CD}_{6} 8^{+}$and

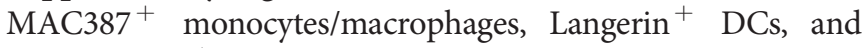
some $\mathrm{CD}_{163}{ }^{+}$monocytes/macrophages (see Supplementary Figure S2 and Table 2). Lymphocytes did not express ACE2 (Table 2). However, we could not detect SARS-CoV genomic RNA by in situ hybridization (ISH) in mucosal immune cells, suggesting that they probably did not support highly productive replication of the virus.

\section{$\mathrm{CD} 163^{+}$monocytes/macrophages were selectively elevated and sequestered virions in intracellular vesicles in the lungs}

Consistent with the upper airway mucosa, IHC analysis revealed significant infiltration of monocytes/macrophages in the lungs at $2 \mathrm{dpi}$, consisting of inflammatory $\left(\mathrm{CD}_{163}{ }^{+}\right)$and newly infiltrated $\left(\mathrm{MAC}_{387^{+}}\right.$) monocytes/macrophages (see Supplementary Figure S3). Importantly, the number of $\mathrm{CD} 63^{+}$cells was about three times higher than that of MAC $387^{+}$population, and these cells were densely gathered around infected alveolar pneumocytes to form cell clusters (Figure 5 and see Supplementary Figure S3). CD163 ${ }^{+}$ monocytes/macrophages within the cell clusters were enlarged in size, suggesting a histological manifestation of activation. Double IHC staining for cell markers and NP or ISH detection of SARS-CoV RNA revealed concentrated signals for both viral NP and RNA in a single intracellular compartment in $\mathrm{CD}_{163}{ }^{+}$and $\mathrm{CD}_{6}{ }^{+}$monocytes/macrophages in the lungs (Figure 5a b,d,e). Furthermore, IHC staining of viral NP, CD81 (cell membrane marker), and CD63 (late endosome marker) in monocytes/macrophages revealed that NP signals were detected within intracellular compartments that were $\mathrm{CD} 81^{+} / \mathrm{CD} 63^{-}$(see Supplementary Figure S4). Because apoptotic bodies are CD63-enriched compartments, ${ }^{21}$ these $\mathrm{NP}^{+}$compartments in monocytes/macrophages were unlikely apoptotic bodies but probably accumulated virions in intracellular vesicles designated as virus-containing compartments (VCCs). ${ }^{22-24}$ Intriguingly, similar VCCs have been recently described in HIV-infected macrophages, which also sequester virions in $\mathrm{CD} 81^{+} / \mathrm{CD} 3^{-}$plasma membrane-derived intracellular invaginations during productive infection. ${ }^{22-24}$ Importantly, although some VCCs are connected to the plasma membrane, they shield viral particles from neutralizing 
Table 1 Detection of SARS-CoV nucleoprotein and RNA in the respiratory mucosa, spleen, and intestine

\begin{tabular}{|c|c|c|c|c|c|c|c|c|c|c|c|c|}
\hline Monkey ID & \multicolumn{4}{|c|}{ Day 2} & \multicolumn{4}{|c|}{ Day 3} & \multicolumn{4}{|c|}{ Day 7} \\
\hline Nasal cavity & - & - & - & - & - & - & - & - & - & + & - & - \\
\hline Oropharynx & + & ++ & + & + & ++ & +++ & ++ & + & ++ & + & ++ & ++ \\
\hline Epiglottis & +++ & ++ & + & + & +++ & + & + & + & - & + & - & - \\
\hline Trachea & + & ++ & + & - & + & ++ & + & + & + & + & - & +++ \\
\hline Left bronchus & + & +++ & + & - & + & + & + & + & + & + & + & + \\
\hline Right bronchus & + & ++ & - & - & - & + & + & ++ & + & + & + & + \\
\hline Left cranial & + & - & + & - & + & - & - & - & - & + & + & - \\
\hline Left middle lobes & - & $++/ R+$ & - & - & - & + & - & - & - & - & - & + \\
\hline Right middle lobes & - & + & - & $+++/ R+$ & - & + & - & - & - & - & - & - \\
\hline Right accessory lobe & + & + & - & $+++/ R+$ & - & + & - & - & - & - & - & $+++/ R+$ \\
\hline Spleen & - & ++ & ++ & - & + & +++ & - & + & + & + & +++ & + \\
\hline Small intestine & ND & ND & ND & ND & ND & ND & ND & ND & + & ++ & ++ & + \\
\hline
\end{tabular}

Abbreviations: ND, not done; SARS-CoV, severe acute respiratory syndrome coronavirus. Tissue samples were tested for SARS-CoV nucleoprotein and viral RNA by immunohistochemistry and in situ hybridization, respectively. The results of staining for SARS-CoV protein were quantified as follows: no positive cells = negative; $1-10$ positive cells per section $=+; 10-50$ positive cells per $\times 10$ field $=++;>50$ positive cells per $\times 10$ field $=+++$. Tissue samples that were positive for viral RNA are indicated as $\mathrm{R}+$. The rest of the tissue samples were negative for viral RNA.

antibodies, raising an obstacle for the elimination of this viral reservoir. $^{23}$ These results suggested that SARS-CoV was probably sequestered into plasma membrane-derived VCCs of monocytes/macrophages after infection. As for DCs, NP signals but not RNA signals were observed in LCs in the lungs (Figure 5c,f and Table 2), indicating virus infection but inefficient productive replication in these cells.

Therefore, we conclude that, following intranasal inoculation, SARS-CoV infects epithelial cells, tissue resident macrophages (CD68), and Langerhans cells (Langerin ${ }^{+}$), which induce the recruitment of monocytes/macrophages $\left(\mathrm{MAC} 387^{+}\right.$and $\mathrm{CD} 163^{+}$) to the site of inflammation and lead to further spread of the virus to $\mathrm{CD} 163^{+}$monocytes/macrophages. The viral targeting of monocytes/macrophages and LCs, primarily antigen-presenting cells (APCs), and in particular the sequestration of virions in an internal niche of monocytes/macrophages and limited virus replication in LCs, may indicate that these cells are possible viral reservoirs that allow SARS-CoV to evade local innate immunity at the primary infection site. Subsequent migration of these infected APCs into draining lymph tissues may lead to systemic dissemination of the infection.

\section{Monocytes/macrophages and Langerhans cells disseminated SARS-CoV into deeper mucosa and established productive infection in tonsils}

To determine the spreading routes of virus-infected APCs, we first examined the lymph tissues that are associated with the primary site of infection, the Waldeyer's tonsilar ring, which is comprised of nasopharyngeal, paired tubal, paired palatine, and lingual tonsils. As shown in Figure 6, numerous $\mathrm{NP}^{+}$cells were detected around the trabeculae septum in tonsils as early as 2 dpi, with morphology suggestive of APCs (Figure 6a). To determine the identity of these cells, we stained $\mathrm{NP}^{+}$sections for CD3 (T cells), CD20 (B cells), DC-SIGN (monocyte-derived DCs (MDDCs)), Langerin (Langerhans cells), and CD68, CD163, and MAC387 (monocytes/macrophages). As shown in Figure 6, there were significantly increased numbers of $\mathrm{CD} 63^{+}$and $\mathrm{CD}^{+} 8^{+}$monocytes/macrophages and Langerhans cells around the trabeculae and T-cell zone in tonsils within $2 \mathrm{dpi}$. Moreover, the majority of LCs (Figure 6b), some CD163 and CD68 (Figure 6c,d), are positive for NP, indicating the migration of $\mathrm{NP}^{+}$monocytes/macrophages and DCs from the primary infection site to lymphoid tissues within 2 dpi. None of the $\mathrm{NP}^{+}$cells expressed CD3, CD20, or MAC387.

Further ISH staining identified virus genome $\mathrm{RNA}^{+}$cells in the tonsil (Figure 6e). Interestingly, although we were not able to detect virus RNA signals in the monocytes/macrophages in the upper airway mucosa, intermediate-to-strong virus RNA signals were observed in many $\mathrm{CD}^{+}{ }^{+}$cells in the tonsils (Figure 6f). Therefore, we conclude that infectious viruses probably reached the deep mucosa and MALT and established productive infection in the tonsils. Importantly, viral antigens were concentrated in Langerin ${ }^{+}$DCs and $\mathrm{CD}_{163}{ }^{+}$and $\mathrm{CD} 8^{+}$monocytes/macrophages. These cells, therefore, are probably vehicles for spreading virus into deeper MALTs 


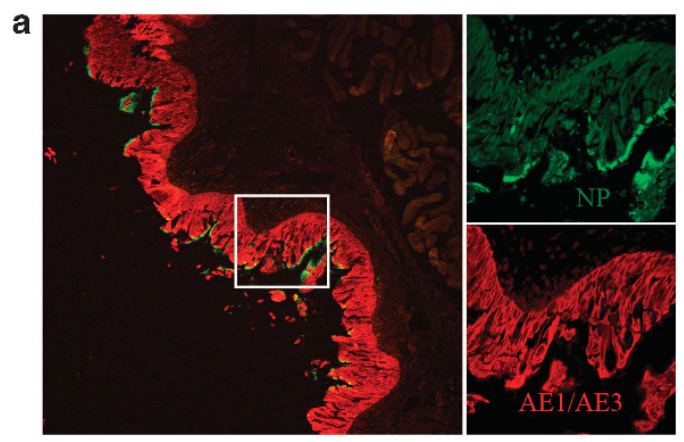

b
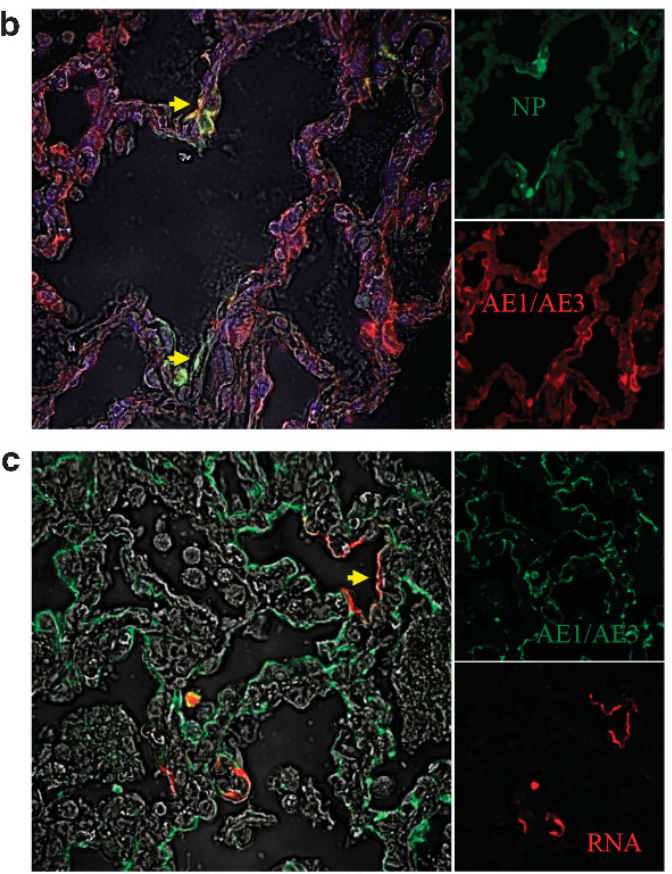

Figure 3 Epithelial cells are targets of severe acute respiratory syndrome coronavirus (SARS-CoV). Tissue sections were double-stained for (a, b) SARS-CoV nucleoprotein (fluorescein isothiocyanate (FITC)) and epithelial membrane antigen (tetramethylrhodamine (TRITC)) (AE1/AE3) or (c) SARS-CoV RNA (TRITC) by in situ hybridization and epithelial membrane antigen (FITC) (AE1/AE3). Left panel shows a lowmagnification overview. Right panel shows single colors from left panel in b and $\mathbf{c}$, and a single color and higher magnification of the boxed area in left panel in a. These sections are representative samples of viral antigen (nucleoprotein)-positive cells that are epithelial cells (a, left $\times 100$, right, $\times 400)$ and type I pneumocytes (b, $\times 630$, yellow arrow) and viral $\mathrm{RNA}^{+}$cells that are type I pneumocytes $(\mathbf{c}, \times 630$, yellow arrow).

shortly ( $2 \mathrm{~d})$ after intranasal inoculation and may contribute to the systemic dissemination of infection.

\section{Viral dissemination into draining lymph nodes (dLNs) through the lymphatics}

The spreading of $\mathrm{NP}^{+}$and viral $\mathrm{RNA}^{+}$cells into tonsils and MALT led us to investigate the route and time course of viral dissemination into draining nodes, which is critical for systemic infection of the immune system. In both human and macaques, lymphatic drainage to the cervical $\mathrm{LN}$ occurs from the nasopharynx, oropharynx, and Waldeyer's ring. Trachea and lung lymphatics drain to the tracheobronchial and hilar
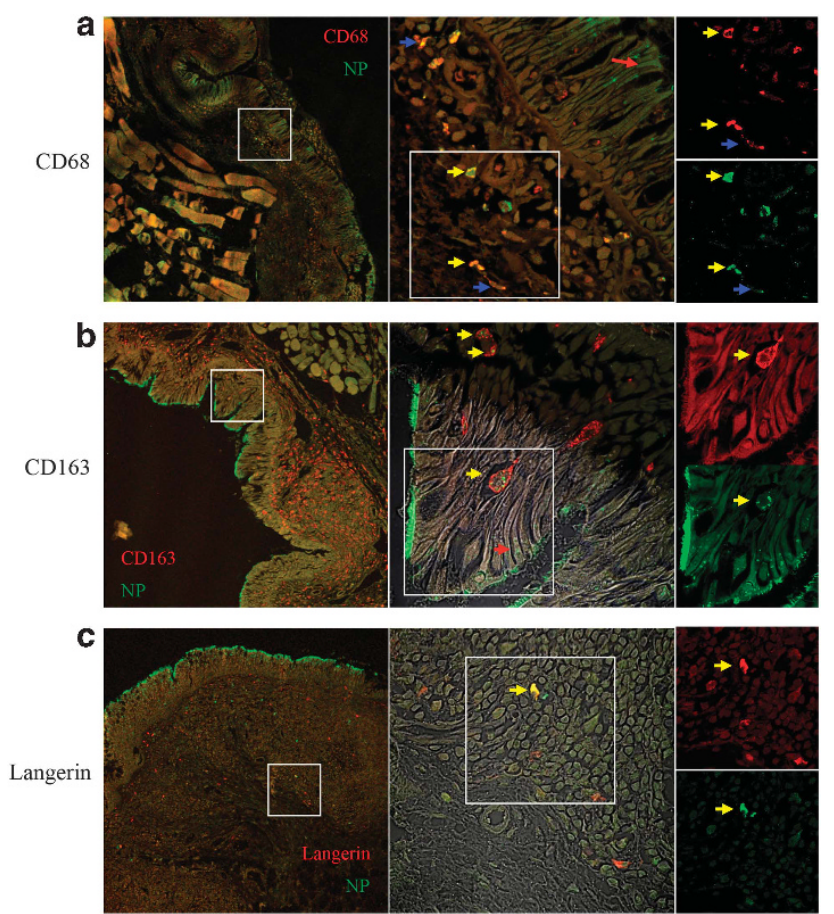

Figure $4 \mathrm{CD}_{163^{+}}$and $\mathrm{CD} 68^{+}$monocytes/macrophages and Langerhans cells in the upper respiratory tract are virus antigen-positive. $(\mathbf{a}-\mathbf{c})$ These sections are representative samples of viral antigen (nucleoprotein (NP))-positive signals that are in the epithelium (a-c, red arrows), the cytoplasm or the intracellular compartment of $\mathrm{CD}^{+} 8^{+}$cells (a, yellow arrows and blue arrow), and the intracellular compartment of $\mathrm{CD} 163^{+}$cells (b, yellow arrows) and the cytoplasm of Langerin ${ }^{+}$ (c, yellow arrows). Pharyngeal mucosa tissue samples were stained for severe acute respiratory syndrome coronavirus (SARS-CoV) NP (fluorescein isothiocyanate) (a-c), CD68 (tetramethylrhodamine (TRITC)) (a), CD163 (TRITC) (b), and langerin (TRITC) (c). The left panel shows a low magnification overview $(\times 100)$. The middle panel shows a higher magnification of the boxed area in the left panel $(\times 400)$. The right panel shows the single color of the boxed area in the middle panel.

LNs, respectively. To monitor the periphery for viral spread, we also studied spleen samples. By IHC, we found $\mathrm{NP}^{+}$cells in lymphatic tissues of all experimentally infected macaques but not control animals (Figure 7). As early as $2 \mathrm{dpi}$, all dLNs tested contained NP antigen in agreement with a broad range of viral seeding into the entire respiratory mucosal tissues. The appearance of NP antigen in the spleen, however, was less frequent at 2 and 3 dpi, observed only among two and three out of four animals, respectively. At $7 \mathrm{dpi}$, however, all of the animals were positive for NP antigen in the spleen (Figure 7 and Table 1). These results demonstrated that, within 2 days of an intranasal inoculation, infected cells quickly disseminate virus to dLNs. Once there, systemic dissemination occurs shortly thereafter. Further study on intestinal samples showed positive signals for NP in the small intestines of all infected animals at $7 \mathrm{dpi}$ (Figure $7 \mathrm{~g}$ and Table 1), providing additional evidence of viral systemic dissemination within 7 days.

We then performed ISH for SARS-CoV RNA to further confirm viral production and determine the frequency and location of productively infected cells in the dLNs. Strong signal 
for viral genome RNA was detected in the tracheobronchial and hilar LNs (Figure 7e,f). Infected cells were localized to the trabecular and germinal center, as well as the T-cell-dependent, paracortical zone of the dLN (Figure $7 \mathbf{f}$ ). These findings suggested that infected cells migrate to the dLN via the afferent lymphatics and enter the node through the subcapsular sinus. From here, these cells traverse to the T-cell-dependent areas in the paracortical regions of the nodes.

Table 2 Summary of SARS-CoV receptor, NP expression, and genomic RNA in the immune cells of infected macaques

\begin{tabular}{|c|c|c|c|c|c|c|c|c|c|}
\hline \multirow[t]{2}{*}{ Immune cell } & \multirow[t]{2}{*}{ ACE2 staining } & \multicolumn{2}{|c|}{ Upper respiratory tract } & \multicolumn{2}{|c|}{ Tonsils and MALT } & \multicolumn{2}{|c|}{ Lung } & \multicolumn{2}{|c|}{ Draining LNs } \\
\hline & & NP & RNA & NP & RNA & NP & RNA & NP & RNA \\
\hline $\mathrm{MAC} 87^{+}$ & +++ & - & - & - & - & - & - & ND & - \\
\hline CD163 ${ }^{+}$ & + & ++ & - & + & - & + & + & ND & + \\
\hline $\mathrm{CD}^{\circ} 8^{+}$ & +++ & ++ & - & + & + & + & + & ND & + \\
\hline Langerin $^{+}$ & ++ & ++ & - & +++ & - & + & - & ND & - \\
\hline $\mathrm{DC}-\mathrm{SIGN}^{+}$ & ND & NA & NA & - & - & NA & NA & ND & + \\
\hline $\mathrm{CD}^{+}$ & - & - & - & - & - & - & - & ND & - \\
\hline $\mathrm{CD}_{20}^{+}$ & - & - & - & - & - & - & - & ND & - \\
\hline
\end{tabular}

Abbreviations: ACE2, angiotensin-converting enzyme 2; DC-SIGN, dendritic cell-specific intercellular adhesion molecule-3-grabbing non-integrin; LN, Iymph node; MALT, mucosa-associated lymph tissue; NA, no cells detected in the tested tissue; ND, not done; NP, nucleoprotein; SARS-CoV, severe acute respiratory syndrome coronavirus. The results of staining for SARS-CoV, NP, and RNA were quantified as follows: undetectable positive signals $=-; 1-2$ positive cells per 10 cells $=+; 3-7$ positive cells per 10 cells $=++;>7$ positive cells per 10 cells $=+++$.
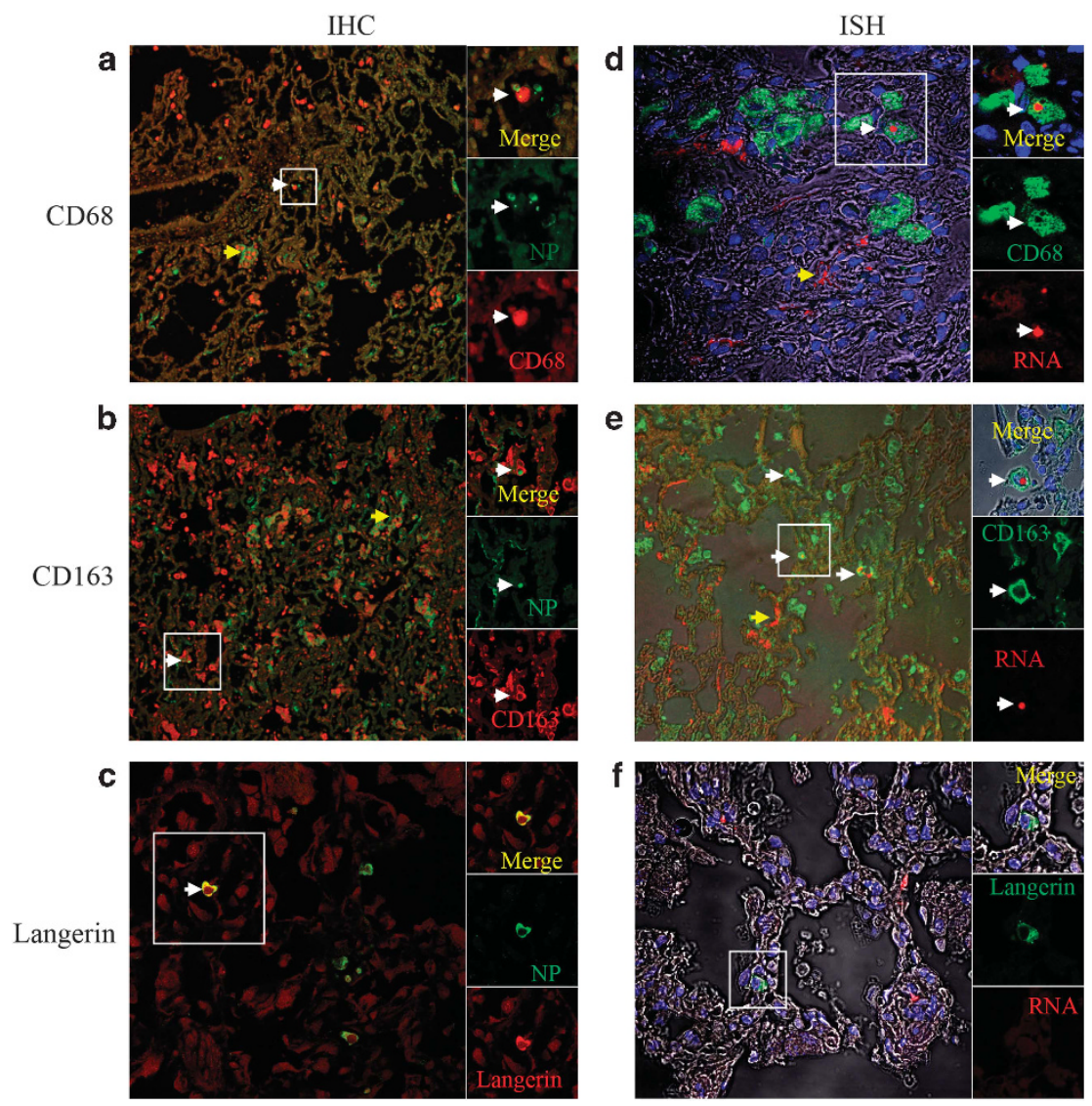

Figure 5 Viral targeting of $\mathrm{CD} 163^{+}$and $\mathrm{CD} 68^{+}$monocytes/macrophages and Langerin ${ }^{+}$cells in the lungs. These sections are representative samples of both viral antigen (nucleoprotein (NP))-positive signals in the intracellular compartment of (a) CD68 ${ }^{+}$(white arrow), (b) CD163 ${ }^{+}$(white arrows), and (c) Langerin $^{+}$(white arrow) cells in the lungs and RNA-positive signals in the intracellular compartment of (d) CD68 ${ }^{+}$(white arrow) and (e) CD163 ${ }^{+}$(white arrows) cells in the lungs. Tissue samples were stained for severe acute respiratory syndrome coronavirus (SARS-CoV) NP (fluorescein isothiocyanate (FITC)) by immunohistochemistry (IHC) (a-c) or (d-f) RNA (tetramethylrhodamine (TRITC)) by in situ hybridization (ISH) and double stained for CD68 (TRITC (a) or FITC (d)), CD163 (TRITC (b) or FITC (e)), and Langerin (TRITC (c) or FITC (f)). In each figure, the left panel shows a low-magnification overview (a and b: $\times 200$, $\mathbf{c}$ and $\mathbf{e}: \times 400$, $\mathbf{d}$ and $\mathbf{f}: \times 630$ ); the right top photo shows a higher magnification of the boxed area in the left panel. The right middle and bottom photo panel show the single colors from the top photo. 

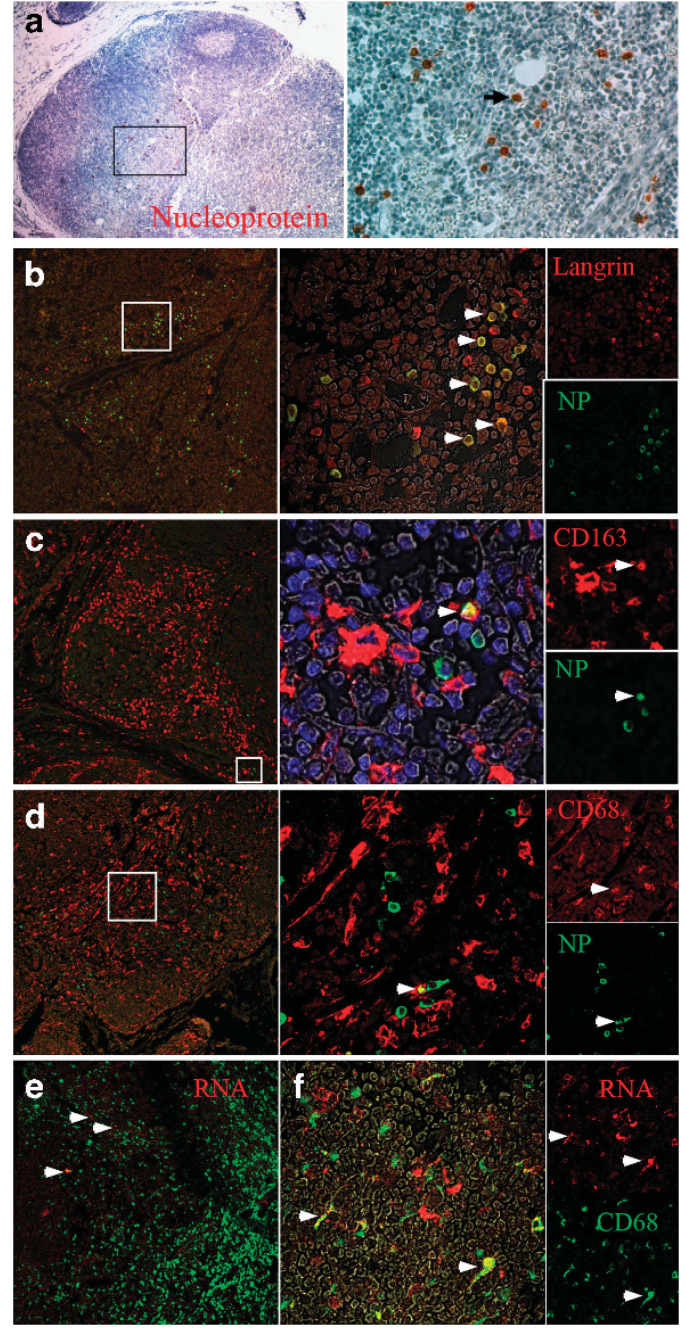

Figure 6 Severe acute respiratory syndrome coronavirus (SARS-CoV) spreads into and establishes productive infection in the tonsils. These sections are representative samples of viral antigen (nucleoprotein

$(\mathrm{NP}))^{+}$and $\mathrm{RNA}^{+}$cells in the tonsils. (a) Viral NP. NP ${ }^{+}$positive cells are indicated by red color and black arrow. (b) Viral NP and Langerin. This section shows that the majority of $\mathrm{NP}^{+}$cells are Langerhans cells $\left(\right.$ Langerin $^{+}$) (white arrow). (c) Viral NP and CD163. This section shows concentrated viral antigen in the cytoplasm of $\mathrm{CD}_{163}{ }^{+}$cells (white arrow). (d) Viral NP and CD68. This section shows viral antigens located in the cytoplasm of $\mathrm{CD}^{+} 8^{+}$cells (white arrow). (e) Viral RNA and CD68. This section shows viral RNA signal around the trabecular and T-cell zones in the tonsils (white arrows). CD68 was used to define the T-cell zone. (f) Viral RNA and CD68. This section showed RNA ${ }^{+}$cells are CD68 (white arrows). Tissue samples are stained for the viral NP (red color) (a) or (fluorescein isothiocyanate (FITC)) (b-d), viral RNA (tetramethylrhodamine (TRITC)) (e, f), Langerin (TRITC) (b), CD163 (TRITC) (c), CD68 (TRITC) (d), and CD68 (FITC) (e, f). The left panel shows a low-magnification overview. The right panel in $\mathbf{a}(\times 40)$ and the middle panel $(\times 630)$ in $\mathbf{b}, \mathbf{c}$, and $\mathbf{d}$ show higher magnification of the boxed area in the left panel. The right panel in $\mathbf{b}, \mathbf{c}, \mathbf{d}$, and $\mathbf{f}$ shows single colors from the middle panel.

$\mathrm{CD} 163^{+}$monocytes/macrophages probably function as a shelter for immune evasion and as vehicles for systemic viral dissemination

We subsequently defined the cell types that carry infectious virus and support productive infection in the hilar lymphoid nodes. dLN sections were studied for RNA production by ISH, together with antibodies for DCs (DC-SIGN and Langerin), monocytes/macrophages (CD68, CD163 and MAC387), T cells (CD3), and B cells (CD20). Consistent with the findings in the tonsils, the increased number of $\mathrm{CD}^{+} 8^{+}$and $\mathrm{CD} 163^{+}$ monocytes/macrophages was readily observed in the dLNs (see Supplementary Figure S5). Compared with the tonsils, only small numbers of LCs were detected in the LNs. Moreover, low-level RNA signals were also found in $\mathrm{CD}^{+}{ }^{+}$cells around the trabecular septum (Figure 8b1, blue arrow) but not in cells expressing MAC387 or Langerin (Figure 8c,d). The strongest RNA signals, however, were observed in DC-SIGN ${ }^{+}$cells in the T-cell-dependent paracortical zone of the hilar LNs (Figure 8a,b2,c). Interestingly, some $\mathrm{DC}-S I G N^{+}$cells with intermediate levels of viral RNA co-expressed CD163 (Figure 8a2, blue arrow). Viral RNA-positive cells in the hilar LNs, therefore, can be summarized as follows: (1) low-level RNA signals in CD68 and DC-SIGN doublepositive cells around the trabecular septum (Figure 8b1, blue arrow), (2) intermediate-level RNA signals in monocyte-like cells with strong $\mathrm{CD}_{163}{ }^{+}$but weak DC-SIGN staining (Figure 8a2, white arrow), (3) DC-like cells stained positive for both CD163 and DC-SIGN (Figure 8a2, blue arrow), and (4) strong RNA signals in DC-like cells with DC-SIGN staining only (Figure 8a1,b2,c, red arrow). DC-SIGN is a MDDC-specific lectin. ${ }^{25}$ These infected cells likely represent different stages of the differentiation of the monocyte subpopulation $\left(\mathrm{CD}^{+} 8^{+}\right.$and $\left.\mathrm{CD} 163^{+}\right)$into DCs, during which monocytes begin to express DC-SIGN and downregulate monocyte marker expression. ${ }^{26,27}$ The increased levels of viral RNA following monocyte differentiation into DCs indicate that viral replication efficiency depends on the differentiation status of the cells. Nevertheless, in comparison with the restricted NP signals in intracellular compartments in $\mathrm{CD}_{6}{ }^{+}$and CD163 ${ }^{+}$ monocytes/macrophages in the upper airway mucosa, strong NP signals were evenly distributed in the cytoplasm of those cells in the tonsils (Figure $\mathbf{6 c}, \mathbf{d}$ ), suggesting that enhanced virus replication was also present in these cells after migration into tonsils.

These results suggested that $\mathrm{CD} 163^{+}$and $\mathrm{CD}^{+} 8^{+}$cells at the primary respiratory mucosa site of infection probably serve as viral reservoirs and allow SARS-CoV to evade local innate immunity by sequestering virus in an internal niche, which is difficult to access from the exterior. Virus replication capacity is subsequently enhanced following the differentiation of monocytes into DCs as they migrate to lymphoid tissue, resulting in productive infection in tonsils and dLNs and eventually systemic dissemination. Notably, in agreement with a previous description that infected macrophages present internally sequestered HIV to uninfected target cells at a virological synapse, ${ }^{28}$ we also observed the formation of infectious synapses between $\mathrm{CD} 63^{+}$cells and $\mathrm{NP}^{+}$cells (see Supplementary Figure S6, blue arrow). Therefore, we conclude that $\mathrm{CD}_{163}{ }^{+}$monocytes/macrophages may have a critical role in the dissemination of infection by sheltering and transferring live virus to the dLNs from the respiratory mucosa 


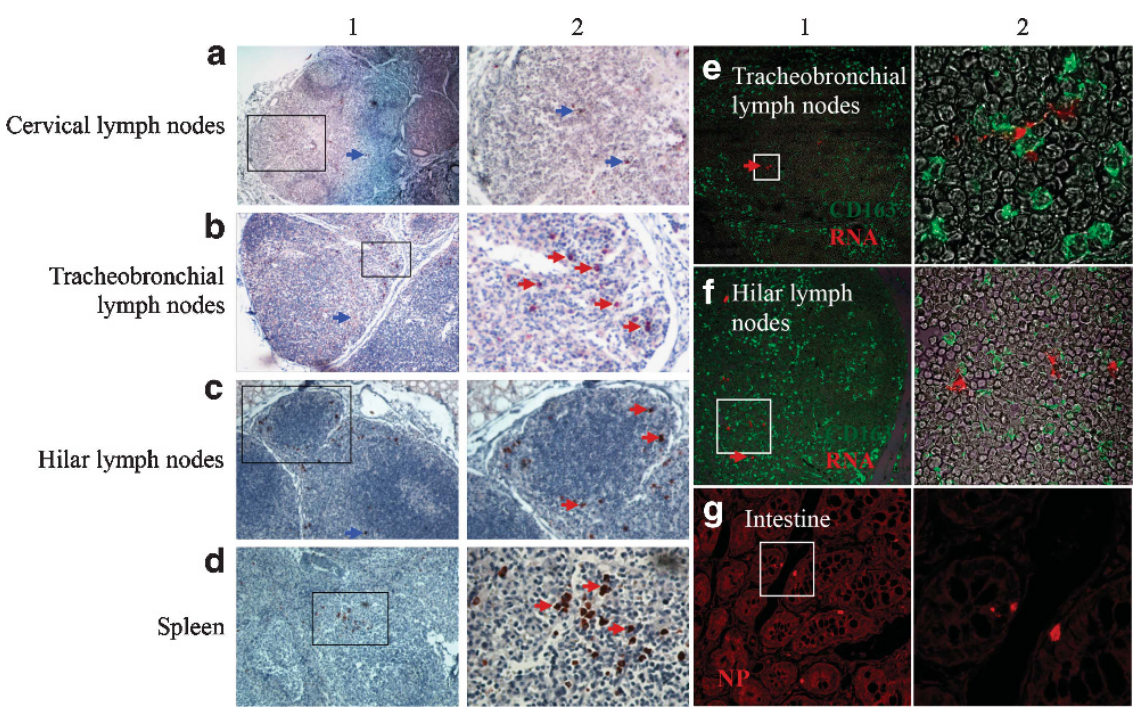

Figure 7 Severe acute respiratory syndrome coronavirus (SARS-CoV) spreads into and establishes productive infection in the draining lymph nodes. (a-d) Representative samples of viral antigen (nucleoprotein (NP)) ${ }^{+}$cells in the draining lymph nodes and spleens. Tissue sections were stained with an anti-NP antibody by immunohistochemistry (IHC). The left panel shows a low-magnification overview. The right panel shows a higher magnification of the boxed area in the left panel. (a) Cervical lymph nodes $(\times 40)$. (b) Tracheobronchial lymph nodes $(\times 40)$. (c) Hilar lymph node $(\times 40)$. (d) Spleen $(\times 200)$. $\mathrm{NP}^{+}$cells were (red color) mostly localized around the trabecular (red arrows), and some are in the T-cell zones in the draining lymph nodes (blue arrows) (a-c). $\mathrm{NP}^{+}$cells were (red color) mostly localized in the red pulp of the spleen (d, red arrow). (e, f) Representative samples of viral RNA ${ }^{+}$cells in the draining lymph nodes. Tissue samples are stained with SARS-CoV RNA (red color) and CD163 (green color) to define the T-cell-dependent region. The left panel shows a low-magnification overview $(\times 100)$. The right panel shows a higher magnification of the boxed area in the left panel $(\mathbf{e} 2: \times 630$ and $\mathbf{f}$ : $\times 400$ ). (e) Tracheobronchial lymph nodes. This section shows infected cells in the lymph nodes (red arrow) (f) Hilar lymph nodes. This section shows infected cells at the subcapsular sinus of the lymph nodes (red arrow). (g) Representative samples of viral antigen (NP) ${ }^{+}$cells in the intestine. Tissue sections were stained with an anti-NP antibody by IHC. The left panel shows a low-magnification overview $(\times 200)$. The right panel shows a higher magnification of the boxed area in the left panel $(\times 630)$.

and further support virus production upon differentiation into MDDCs.

\section{DISCUSSION}

The human mucosa barrier constitutes the first line of host defense against virus transmission and infection. However, many viruses have developed efficient strategies to traverse the mucosal barrier, often by direct invasion of the epithelium. Upon penetration of this barrier, viruses interact with mucosal monocyte/macrophages, lymphocytes, and LCs, which form the second line of innate host defense and bridge the third line of adaptive immunity. ${ }^{6,10}$ Although this interaction is necessary for triggering immune protection, the virus may highjack immune cells to facilitate its dissemination and infection. For example, in the case of HIV sexual transmission, mucosal LCs and DCs have a role in capturing and disseminating newly produced virus to the main target, $\mathrm{CD} 4^{+} \mathrm{T}$ cells, which are essential for the establishment of productive infection. ${ }^{11,29}$ To date, the early respiratory mucosal events of SARS-CoV infection remain incompletely understood. In this study, we investigated these events in Chinese macaques following the intranasal inoculation of a pathogenic live SARS-CoV. This model has been reported to mirror the pathogenesis of human SARS in several studies. ${ }^{19,30,31}$ The clinical relevance of this model includes elevated body temperature, lung pathology, SARS-CoV antigen detection in type I pneumocytes, and eventually viral systemic dissemination in infected macaques, providing strong support for the extrapolation of the findings in our study to the transmission and dissemination of respiratory infection in humans. ${ }^{19,30,31}$

We first examined the process of SARS-CoV infection from the site of entry to the respiratory portion of the lung and subsequent systemic dissemination, as well as the status and role of respiratory mucosa innate immunity in protection, productive infection, and transmission of the virus. By measuring the cells that were positive for viral NP protein, we found that SARS-CoV spread quickly from the upper respiratory tract to the lung within 2 dpi (Figure 2), which includes epithelial cells from the nasopharynx, oropharynx, laryngopharynx, trachea, and bronchus (Figure 2a-g). The majority of positive cells were abundantly identified in the pharynx areas, including the epithelial cells lining the salivary ducts, that we previously described (Figure 2). ${ }^{20}$ These areas are likely one of the major sites of SARS-CoV invasion and shedding. Under the epithelial cells, we found that monocytes/ macrophages and DCs were actively involved in SARS-CoV infection. As shown in Figures 4 and 5, resident $\mathrm{CD} 68^{+}$ macrophages and inflammatory $\mathrm{CD} 163^{+}$macrophages were among the early target cells infected within $2 \mathrm{dpi}$. The infection of epithelial cells resulted in the significant recruitment of circulating blood monocytes, macrophages, and eventually lymphocytes to the sites of viral invasion. In particular, the rapid recruitment of $\mathrm{MAC} 387^{+}$monocytes/macrophages and selective accumulation of $\mathrm{CD}_{163}{ }^{+}$macrophages indicated 

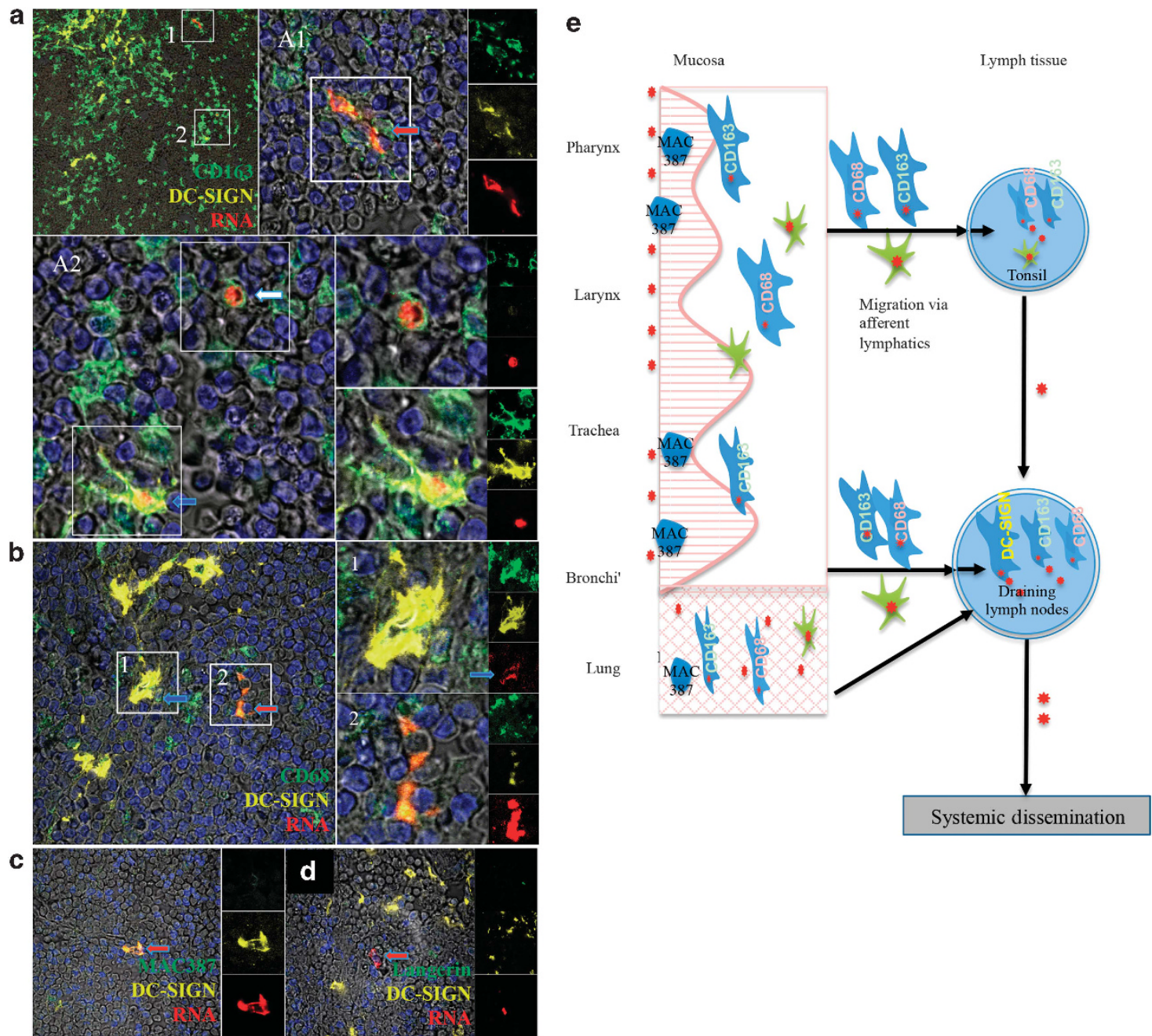

Figure 8 Monocyte-derived dendritic cells are productively infected by severe acute respiratory syndrome coronavirus (SARS-CoV) in the draining lymph nodes. Hilar lymph nodes are stained for SARS-CoV RNA-tetramethylrhodamine, DC-SIGN-yellow, CD163-fluorescein isothiocyanate (FITC), CD68-FITC, MAC387-FITC and Langerin-FITC. The left panel shows a low-magnification overview. The right panel shows a higher magnification of the boxed area in the left panel. (a) Viral RNA, CD163, and DC-SIGN $(\times 100)$. This section is a representative sample of productively infected cells that are DC-SIGN ${ }^{+}$and CD163 $^{-}\left(\mathbf{a} 1, \times 400\right.$, red arrow), CD163 ${ }^{\text {hi }}$ and DC-SIGN ${ }^{\text {low }}(a 2, \times 400$, white arrow $)$ or CD163 ${ }^{\text {hi }}$ and DC-SIGN ${ }^{\text {hi }}$ (a2, blue arrow). (b) Viral RNA, CD68, and DC-SIGN $(\times 400)$. This section shows that infected cells are DC-SIGN ${ }^{+}$and CD68 ${ }^{+}(\mathbf{b} 1$, blue arrow) and DC-SIGN ${ }^{+}$and CD68 ${ }^{-}$(b2, red arrow). (c) Viral RNA, MAC387, and DC-SIGN ( $\left.\times 400\right)$. Infected cells are DC-SIGN ${ }^{+}$and MAC387 ${ }^{-}$(red arrow). (d) Viral RNA, Langerin, and DC-SIGN $(\times 400)$. Infected cells are DC-SIGN ${ }^{+}$and Langerin ${ }^{-}$(red arrow). (e) Model of mucosal transmission of SARS-CoV. Langerhans cells are shown in green, macrophages in blue, and virus in red. SARS-CoV infects the epithelium and resident macrophages shortly after infection. Immune cells are subsequently recruited in droves via a chemotactic gradient from the infected epithelium and/or tissue-resident and alveolar macrophages. Those inflammatory cells are heterogeneously composed of CD $163^{+}$macrophages and recently infiltrated MAC387 monocytes/macrophages. $\mathrm{CD}_{163^{+}}$macrophages are productively infected when fighting against the infection and subsequently transmit virus to the draining lymph nodes by migrating via the afferent lymphatics. In the nasopharynx and oropharynx, Langerhans cells were also infected and migrated to the mucosa-associated lymphoid tissue. However, active viral replication was not detected in the Langerhans cells. Their role in virus production and transmission remains uncertain.

active chemotaxis owing to local inflammation. ${ }^{7}$ However, the monocyte/macrophage population was not homogeneous in its ability to be infected and to support viral replication and transmission (Figures 4 and 5). Despite the universal expression of ACE2 on MAC387 macrophages (see Supplementary Figure S2B), SARS-CoV mainly infected $\mathrm{CD} 163^{+}$or $\mathrm{CD} 68^{+}$ macrophages in the respiratory mucosa (Figures 4 and 5). These findings indicate that, in addition to ACE2 expression, other factors such as the activation/differentiation status of monocytes/macrophages are probably critical for
SARS-CoV infection, and $\mathrm{CD} 163^{+}$macrophages might be crucial for the establishment and dissemination of infection (Figure 8).

Our data suggest that $\mathrm{CD}_{6}{ }^{+}$and $\mathrm{CD} 163^{+}$monocytes/ macrophages may serve as shelters for immune evasion and vehicles for productive infection and dissemination of the virus into MALT (e.g., tonsils) and dLNs (Figures 6 and 8). Several previous studies showed that in vitro infection of primary human monocyte-derived macrophages/DCs was likely abortive. ${ }^{32,33}$ As these studies used monocyte-derived primary 
macrophages/DCs, mainly terminally differentiated cells, they might not reflect the in vivo situation, especially when monocytes/macrophages are highly plastic and heterogeneous in vivo. We detected compartmented viral RNA in VCCs of monocytes/macrophages in respiratory mucosa but abundant viral RNA in the cytoplasm of MDDCs in the dLNs (Figure 8). We speculated that, although infected monocytes/macrophages might not produce viruses effectively in mucosa, they might have supported a low level of viral replication after they differentiated into DCs in the dLNs. This is possible because cytokines (e.g., tumor necrosis factor) produced by infected cells could skew cell differentiation from macrophages to DCs. ${ }^{34,35}$ To this end, low levels of SARS-CoV replication were found in MDDCs in one study. ${ }^{36}$ Critically, infectious SARS$\mathrm{CoV}$ was released into culture supernatants and recovered from infected MDDCs. ${ }^{36}$ Recent studies also demonstrated that SARS-CoV infection of monocytes/macrophages could induce DC-SIGN expression on these cells. ${ }^{37} \mathrm{CD} 163$ protein levels are suppressed when monocytes differentiate toward DCs. ${ }^{27}$ To determine the activation/differentiation status of infected monocytes/macrophages, we measured the expression of DC-SIGN and CD163 on viral $\mathrm{RNA}^{+}$cells in dLNs (Figure 8). Interestingly, we likely identified virus-infected monocytes at different stages of differentiation into DCs in the dLNs, as indicated by strong RNA signals in MDDCs $\left(\mathrm{DC} \mathrm{SIGN}^{+}\right)$, which still express low levels of CD163 (Figure 8). The lack of active viral replication in mucosal monocytes/macrophages and the restriction of virions in the $\mathrm{CD} 81^{+} / \mathrm{CD} 3^{-}$intracellular compartment (see Supplementary Figure S4) are probably beneficial for viruses to escape local antiviral innate immunity, because low levels of viral RNA in the cytoplasm and protein on the cell surface help the virus to avoid Toll-like receptor sensing and killing by natural killer cells. This hypothesis is based on similar intracellular structures recently described in HIV-infected macrophages, which concentrate virions in $\mathrm{CD} 81^{+}$, plasma membrane-derived intracellular invaginations during infection. ${ }^{22,24}$ Importantly, the sequestered HIV was able to translocate to the virological synapse formed between infected macrophages and uninfected T cells. ${ }^{28}$ Consistently, we also observed virological synapses formed between $\mathrm{CD}_{163}{ }^{+}$monocytes and $\mathrm{NP}^{+}$cells (see Supplementary Figure S6), suggesting possible cell-to-cell transmission. Moreover, the increased virus production efficiency in monocytes upon differentiation into DCs might have resulted in the establishment of productive infection in lymphoid tissue, virus release into the circulating system, and eventual systemic dissemination.

In addition to monocytes/macrophages, intraepithelial or subepithelial LCs may also contribute to the establishment of systemic infection via the dissemination of newly produced virus or the presentation of infectious virus to other cells. From the mucosal site, LCs migrate into the afferent lymphatics, enter the LNs through the subcapsular sinus, and travel toward the $\mathrm{T}$-cell areas in the paracortical regions. LCs specifically express Langerin and were previously reported to mediate the transmission of HIV-1 to T cells and to keep LCs refractory to HIV-1 transmission in different studies. ${ }^{11,29}$ To determine the role of LCs in SARS-CoV transmission, we double stained pharyngeal mucosal tissue sections with antibodies against SARS NP and DC-SIGN or Langerin. We revealed the distribution, migration, and potential role of LCs in the upper respiratory system during SARS-CoV infection, as schematically summarized in Figure 8. We readily found a large number of LCs in the intraepithelial and subepithelial areas of the pharynx (Figure 4c and see Supplementary Figure S1A). Similar to monocytes/macrophages, upon encountering a virus, $\mathrm{NP}^{+}$LCs were found in the epithelial lining of the pharyngeal mucosa (Figure 4c) and migrated rapidly from the pharynx mucosa into the tonsils (Figure $6 \mathbf{b}$ ). The large number of $\mathrm{NP}^{+}$ LCs in the tonsil suggested a highly effective process by LCs for antigen presentation and virus dissemination into lymphoid tissues (Figure 6b). The lack of viral RNA signal in LCs by ISH, however, indicated that LCs probably do not support productive viral replication. We, however, cannot exclude the possibility that $\mathrm{NP}^{+}$LCs are able to transmit virus to other cells and produce small amounts of virus. To this end, we detected many cells harboring viral RNA in the tonsils by ISH, which confirmed virus infection of the upper respiratory mucosa (Figures 6 and 7). This finding indicated that tonsils can be another source of virus in oral swabs, particularly early in infection. To our knowledge, this is the first report of productive SARS-CoV infection in the tonsils. SARS-CoV production preferentially persists in the upper respiratory mucosa during the acute phase, as indicated by the prevalent virus antigen in the mucosal epithelium and immune cells beneath the epithelium (Figure 2), and more importantly, the persistent viral loads in the oral swabs (Figure 1).

Type I pneumocytes are the major early target cells in the lung for SARS-CoV infection in Chinese macaques, similar to humans who died of SARS. ${ }^{4}$ Under our experimental conditions, which involved a large viral inoculum, NP signal was readily detected in alveolar pneumocytes in macaques (Figure 2). Among four macaques killed at $2 \mathrm{dpi}$, however, only two (AD0506 and AD0508) exhibited extensive positive signals (Figure 2), and only scattered $\mathrm{NP}^{+}$cells were found in the lungs of two other macaques. These results, together with the $\mathrm{NP}^{+}$cells found in the LNs draining the lungs, suggested that SARS-CoV was disseminated to the lower respiratory system in a rather effective and rapid manner. Moreover, viral RNA was readily detected in type I pneumocytes, which was indicative of productive infection established in the lungs. $\mathrm{T}$ cells were productively infected in the LNs of patients who died of SARS. ${ }^{16}$ We, however, could not detect infected T cells in the respiratory mucosa or LNs during the acute phase ( $2 \mathrm{dpi}$ ) (data not shown). $\mathrm{T}$ cells, therefore, are unlikely to be early targets of infection in Chinese macaque model. Whether these findings underlie the lack of SARS in Chinese macaques remains to be investigated in future. ${ }^{18}$

In summary, our findings led to a model of SARS-CoV mucosal infection (Figure 8). The virus breaches the mucosal barrier by infecting the respiratory epithelium, mainly around the pharynx and larynx, and subsequently infects mucosal 
Langerhans cells and resident macrophages. In the meantime, $\mathrm{CD} 63^{+}$and $\mathrm{MAC}_{38} 7^{+}$monocytes/macrophages are quickly recruited in droves by a chemotactic gradient from infected epithelium and tissue-resident macrophages. However, these monocytes/macrophages are unlikely to be sufficient to eliminate the incoming viral attack, which allows LGs and $\mathrm{CD} 63^{+} / \mathrm{CD} 68^{+}$monocytes/macrophages carrying the virus to migrate to the MALT and dLNs via the afferent lymphatics. Once inside, $\mathrm{CD} 163^{+}$monocytes/macrophages unexpectedly support productive viral replication upon differentiation into DCs. Free virus and infected DCs may infect or trans-infect new target cells, respectively, and then leave through the efferent lymphatics for systemic dissemination (e.g., spleen and intestine, Figure 7d,g).

\section{METHODS}

Animals, tissues, and SARS-CoV infection. SARS-CoV-infected animals and control animals were all adult female rhesus monkeys (Chinese rhesus macaques). A total of 12 Chinese rhesus macaques were challenged with live pathogenic SARS-CoV (PUMC01, $\left.\mathrm{TCID}_{50}=50\right)$ via the nasal cavity route as previously described. ${ }^{20}$ An additional three uninfected macaques were inoculated with phosphate-buffered saline as negative controls. Animals were subjected to daily measurement of anal temperature, routine blood assays, and chest radiography. Four animals were killed at 2, 3, and 7 dpi (Figure 1). All animals were humanely euthanized with an intravenous overdose of pentobarbital and were immediately necropsied. A complete set of tissues, including multiple levels of the respiratory tract, dLNs, and spleens, was collected. The respiratory tract includes the nasal cavity, nasopharynx, oropharynx, epiglottis, larynx, trachea, left bronchus, right bronchus, and lungs (left and right cranial, middle and caudal lobes, and right accessory lobe). Tissues were fixed in $10 \%$ neutral buffered formalin, embedded in paraffin, and sectioned at $5 \mu \mathrm{m}$. The experiment was conducted in our Bio-Safety Level 3 animal facility. The animal protocols were approved by our institutional animal welfare committee.

Nested RT-PCR. Pharyngeal swab samples were collected from infected monkeys starting on the first dpi. Chinese macaques were killed on days 2, 3, and 7 after virus challenge, and lung homogenates of each animal were generated. To detect the viral RNA in specimens of SARS-CoV-inoculated monkeys, total RNA was extracted from various specimens with TRIZOL (Invitrogen, Grand Island, NY) and tested for SARS-CoV by nested RT-PCR as previously described. ${ }^{18,19}$ Briefly, RT-PCR was performed in a $50-\mu$ l reaction volume with an outer primer pair ( $5^{\prime}$-GCTGCATTGGTTTGTTATATCGTTATGC- ${ }^{\prime}$ and $5^{\prime}$-ATACAGAATACATAGATTGCTGTTATCC- $3^{\prime}$ ) and an inner primer pair $\left(5^{\prime}\right.$-TCACTTGCTTCCGTTGAGGTAGCAGCGT GGTGGTTCATACAA- $3^{\prime}$ and $5^{\prime}$-GGTTTCGGATGTTACAGCGT CTCCCGGCAGAAAGCTGTAAGCT-3'). All PCR products were verified by nucleotide sequencing.

IHC staining of paraffin sections. IHC staining was performed on 5 - $\mu \mathrm{m}$ paraffin sections of formalin-fixed tissue samples. Samples were deparaffinized and rehydrated. After blocking endogenous peroxidases with $0.3 \% \mathrm{H}_{2} \mathrm{O}_{2}$ and $0.03 \% \mathrm{NaN}_{3}$ for $30 \mathrm{~min}, 10 \%$ normal goat serum was applied for $30 \mathrm{~min}$ at room temperature. Rabbit anti-SARS $\mathrm{CoV}$ nucleocapsid antibody was added at room temperature for $1 \mathrm{~h}$. Sections were then washed and incubated with peroxidase-conjugated goat anti-rabbit immunoglobulin G antibody (DakoCytomation, Glostrup, Denmark) for $1 \mathrm{~h}$ at room temperature. The signal was developed with diaminobenzidine chromogen substrate (DakoCytomation). Sections were counterstained with Mayer's hematoxylin (DakoCytomation) and mounted with Crystal mounting. Electronic images were visualized under a microscope (Eclipse E600;
Nikon, Melville, NY), were captured, and saved to a computer using the software ACT-1 (Nikon).

Double immunofluorescence staining of paraffin sections. Immunofluorescence staining was performed on $5-\mu \mathrm{m}$ paraffin sections of formalin-fixed tissue samples. Samples were deparaffinized and rehydrated. After blocking with normal goat serum for $30 \mathrm{~min}$ at room temperature, rabbit anti-SARS $\mathrm{CoV}$ nucleocapsid antibody was applied at $4{ }^{\circ} \mathrm{C}$ overnight or for $1 \mathrm{~h}$ at room temperature, followed by Alex 488-conjugated goat anti-rabbit immunoglobulin $\mathrm{G}$ antibody for $1 \mathrm{~h}$ at room temperature. Additional immunofluorescence staining was then performed by incubating the second set of primary antibodies for $1 \mathrm{~h}$ at room temperature or $4{ }^{\circ} \mathrm{C}$ overnight, followed by the detection of signals with appropriate fluorescent secondary antibodies for $1 \mathrm{~h}$ at room temperature.

ISH studies. ISH was developed for the detection of SARS-CoV. DNA fragments for the M, N protein and spike protein from the SARS-CoV strain from Hong Kong (AY278491) were used as templates to generate an RNA probe. The probe was labeled with digoxigenin. Formalin-fixed, paraffin wax-embedded tissues were cut into $5-\mu \mathrm{m}$ sections. After deparaffinization and rehydration, the sections were treated with microwave heating in an $800-\mathrm{W}$ domestic microwave oven on medium power for $10 \mathrm{~min}$ in $0.01 \mathrm{~m}$ sodium citrate buffer. Hybridization was performed at high stringency with $150 \mathrm{ng} \mathrm{ml}^{-1}$ denatured probe at $45^{\circ} \mathrm{C}$ for $16 \mathrm{~h}$ in $50 \mu \mathrm{l}$ of hybridization mix in a moist chamber. ${ }^{20}$ Excess probe was removed by washing twice in $2 \times$ SSC for $20 \mathrm{~min}$ at $45^{\circ} \mathrm{C}$. This was followed by two washes in $1 \times$ SSC and $0.1 \times$ SSC for $20 \mathrm{~min}$ at $45^{\circ} \mathrm{C}$. Immunological detection was conducted using a DIG Nuclear Acid Detection Kit (Roche, Basel, Switzerland) following the manufacturer's instructions. Briefly, after incubation with $1 \%$ blocking reagent for $30 \mathrm{~min}$, the sections were incubated with sheep anti-digoxigenin alkaline phosphatase conjugate diluted 1:500 for $2 \mathrm{~h}$ at room temperature. After three separate washes with washing buffer, ${ }^{20}$ the signal was developed using $200 \mu \mathrm{l} \mathrm{HNPP/}$ Fast Red TR mix in the dark for $45 \mathrm{~min}$. Sections were counterstained with 4,6-diamidino-2-phenylindole. Tissue sections incubated with a sense RNA probe were used as negative controls.

Immunofluorescence-fluorescence in-situ hybridization studies. Cytokeratin (AE1/AE3, Dako, 1:50) was used as an epithelial marker, while Mac387, CD163, and CD68 (Dako, 1:20) were used as macrophage markers, DC-SIGN as a DC marker, Langerin as a Langerhans cell marker, CD3 as a T cell marker, and CD20 as a B cell marker. Double immunofluorescence-fluorescence in-situ hybridization was performed as for ISH, with additional immunofluorescence detection performed by incubating primary antibodies overnight before the detection of signals with the appropriate fluorescent secondary antibodies. ISH signals were detected using anti-digoxigenin alkaline phosphatase conjugate and HNPP/Fast Red TR mix (Roche). The secondary antibody for immunofluorescence was generally anti-mouse fluorescein thiocyanate (Invitrogen). Nuclei were counterstained with 4,6-diamidino-2-phenylindole in anti-fade mounting medium (Vectashield, Vector Laboratories, Burlingame, CA).

Confocal microscopy. Confocal microscopy was performed using a Carl Zeiss LSM 510 META confocal microscope (Oberkochen, Germany) equipped with four lasers. Adobe Photoshop (version 7.0; Adobe, San Jose, CA) was used to assign colors to the four channels collected: Alexa 568 and HNPP/Fast Red, whose fluoresce when exposed to a 568-nm wavelength laser appear red; Alexa 488 (Molecular Probes, Grand Island, NY) appears green; and Alexa 633 (Molecular Probes) appears yellow. The four channels were collected simultaneously. In some tissues and to differentiate between individual cells, Hoechst 33258 (nuclear marker, Molecular Probes) was used at $1 \mu \mathrm{g} \mathrm{ml}^{-1}$ and incubated for $5 \mathrm{~min}$, and tissues were then rinsed in water. Co-localization of antigens was demonstrated by the addition of colors as indicated in the figure legend. 
SUPPLEMENTARY MATERIAL is linked to the online version of the paper at http://www.nature.com/mi

\section{ACKNOWLEDGMENTS}

We thank the US NIH National Heart, Lung and Blood Institute (RO1 HL080211-02 to Z.C.), CRF (HKU5/CRF/13G), the TNPRC base grant RR00164, and University Development Fund/Li Ka Shing Faculty of Medicine Matching Fund of the University of Hong Kong to its AIDS Institute for financial support. We thank D.D. Ho, M. Chan, K. Liu, and K.Y. Yuen for support and scientific discussions.

\section{AUTHOR CONTRIBUTIONS}

Z.C., Q.W., and L.L. designed the study. L.L. and Q.W. analyzed the data. L.L. and Z.C. contributed to writing the paper. Z.C. is the principal investigator, with overall responsibility for the design of the study and writing of the report. Q.W. and C.Q. coordinated the autopsy organization and collection of autopsy materials of Chinese macaques. Q.W. was involved in the development of the RT-PCR assay. L.L., X.A., K.N., H.W., C.M., J.P., and A.L. were involved in the development and performance of the SARS-CoV IHC and ISH assays. All authors were involved in the correlative interpretation of the pathological and molecular data.

\section{DISCLOSURE}

The authors declare no conflict of interest.

(c) 2016 Society for Mucosal Immunology

\section{REFERENCES}

1. Drosten, C. et al. Identification of a novel coronavirus in patients with severe acute respiratory syndrome. N. Engl. J. Med. 348, 1967-1976 (2003).

2. Buchholz, U. et al. Contact investigation of a case of human novel coronavirus infection treated in a German hospital, October-November 2012. Eurosurveillance 18, 1-7 (2013).

3. Zaki, A.M., van Boheemen, S., Bestebroer, T.M., Osterhaus, A.D. \& Fouchier, R.A. Isolation of a novel coronavirus from a man with pneumonia in Saudi Arabia. N. Engl. J. Med. 367, 1814-1820 (2012).

4. Nicholls, J.M. et al. Time course and cellular localization of SARS-CoV nucleoprotein and RNA in lungs from fatal cases of SARS. PLoS Med. 3 e27 (2006).

5. Richt, J.A. et al. Recently emerged swine influenza A virus (H2N3) causes severe pneumonia in Cynomolgus macaques. PLoS One 7, e39990 (2012).

6. Thibeault, S.L., Rees, L., Pazmany, L. \& Birchall, M.A. At the crossroads: mucosal immunology of the larynx. Mucosal Immunol. 2, 122-128 (2009).

7. Bergthaler, A. et al. Viral replicative capacity is the primary determinant of lymphocytic choriomeningitis virus persistence and immunosuppression. Proc. Natl. Acad. Sci. USA 107, 21641-21646 (2010).

8. Matloubian, M., Kolhekar, S.R., Somasundaram, T. \& Ahmed, R. Molecular determinants of macrophage tropism and viral persistence: importance of single amino acid changes in the polymerase and glycoprotein of lymphocytic choriomeningitis virus. J. Virol. 67, 7340-7349 (1993).

9. Norris, B.A., Uebelhoer, L.S., Nakaya, H.I., Price, A.A., Grakoui, A. \& Pulendran, B. Chronic but not acute virus infection induces sustained expansion of myeloid suppressor cell numbers that inhibit viral-specific T cell immunity. Immunity 38, 309-321 (2013).

10. Hickman, H.D. et al. Anatomically restricted synergistic antiviral activities of innate and adaptive immune cells in the skin. Cell Host Microbe 13, 155-168 (2013).

11. Saba, E. et al. HIV-1 sexual transmission: early events of HIV-1 infection of human cervico-vaginal tissue in an optimized ex vivo model. Mucosal Immunol. 3, 280-290 (2010).

12. To, K.F. et al. Tissue and cellular tropism of the coronavirus associated with severe acute respiratory syndrome: an in-situ hybridization study of fatal cases. J. Pathol. 202, 157-163 (2004).

13. Peiris, J.S. et al. Clinical progression and viral load in a community outbreak of coronavirus-associated SARS pneumonia: a prospective study. Lancet 361, 1767-1772 (2003).
14. Ding, Y. et al. The clinical pathology of severe acute respiratory syndrome (SARS): a report from China. J. Pathol. 200, 282-289 (2003).

15. Wu, V.C. et al. Acute renal failure in SARS patients: more than rhabdomyolysis. Nephrol. Dial. Transplant. 19, 3180-3182 (2004).

16. Gu, J. et al. Multiple organ infection and the pathogenesis of SARS. J. Exp. Med. 202, 415-424 (2005).

17. Farcas, G.A. et al. Fatal severe acute respiratory syndrome is associated with multiorgan involvement by coronavirus. J. Infect. Dis. 191, 193-197 (2005).

18. Chen, Y. et al. Rhesus angiotensin converting enzyme 2 supports entry of severe acute respiratory syndrome coronavirus in Chinese macaques. Virology 381, 89-97 (2008).

19. Qin, C. et al. An animal model of SARS produced by infection of Macaca mulatta with SARS coronavirus. J. Pathol. 206, 251-259 (2005).

20. Liu, L. et al. Epithelial cells lining salivary gland ducts are early target cells of severe acute respiratory syndrome coronavirus infection in the upper respiratory tracts of rhesus macaques. J. Virol. 85, 4025-4030 (2011).

21. Crescitelli, R. et al. Distinct RNA profiles in subpopulations of extracellular vesicles: apoptotic bodies, microvesicles and exosomes. J. Extracell. Vesicles 2, 20677-20686 (2013).

22. Deneka, M., Pelchen-Matthews, A., Byland, R., Ruiz-Mateos, E. \& Marsh, M. In macrophages, HIV-1 assembles into an intracellular plasma membrane domain containing the tetraspanins CD81, CD9, and CD53. J. Cell. Biol. 177, 329-341 (2007).

23. Koppensteiner, H., Banning, C., Schneider, C., Hohenberg, H. \& Schindler, M. Macrophage internal HIV-1 is protected from neutralizing antibodies. J. Virol. 86, 2826-2836 (2012).

24. Welsch, S., Keppler, O.T., Habermann, A., Allespach, I., Krijnse-Locker, J. \& Krausslich, H.G. HIV-1 buds predominantly at the plasma membrane of primary human macrophages. PLoS Pathog. 3, e36 (2007).

25. Geijtenbeek, T.B. et al. Identification of DC-SIGN, a novel dendritic cellspecific ICAM-3 receptor that supports primary immune responses. Cell 100, 575-585 (2000).

26. Sulahian, T.H. et al. Human monocytes express CD163, which is upregulated by IL-10 and identical to p155. Cytokine 12, 1312-1321 (2000).

27. Buechler, C., Ritter, M., Orso, E., Langmann, T., Klucken, J. \& Schmitz, G Regulation of scavenger receptor CD163 expression in human monocytes and macrophages by pro- and antiinflammatory stimuli. J. Leukoc. Biol. 67, 97-103 (2000).

28. Gousset, K. etal. Real-time visualization of HIV-1 GAG trafficking in infected macrophages. PLoS Pathog. 4, e1000015 (2008).

29. Hladik, F. et al. Initial events in establishing vaginal entry and infec tion by human immunodeficiency virus type-1. Immunity 26, 257-270 (2007).

30. Li, B.J. et al. Using siRNA in prophylactic and therapeutic regimens against SARS coronavirus in Rhesus macaque. Nat. Med. 11, 944-951 (2005).

31. Lawler, J.V. et al. Cynomolgus macaque as an animal model for severe acute respiratory syndrome. PLoS Med. 3, e149 (2006).

32. Law, H.K. et al. Chemokine up-regulation in SARS-coronavirus-infected, monocyte-derived human dendritic cells. Blood 106, 2366-2374 (2005).

33. Cheung, C.Y. et al. Cytokine responses in severe acute respiratory syndrome coronavirus-infected macrophages in vitro: possible relevance to pathogenesis. J. Virol. 79, 7819-7826 (2005).

34. Rivollier, A., He, J., Kole, A., Valatas, V. \& Kelsall, B.L. Inflammation switches the differentiation program of Ly6Chi monocytes from antiinflammatory macrophages to inflammatory dendritic cells in the colon. J. Exp. Med. 209, 139-155 (2012).

35. Chomarat, P., Dantin, C., Bennett, L., Banchereau, J. \& Palucka, A.K. TNF skews monocyte differentiation from macrophages to dendritic cells. J. Immunol. 171, 2262-2269 (2003).

36. Spiegel, M., Schneider, K., Weber, F., Weidmann, M. \& Hufert, F.T. Interaction of severe acute respiratory syndrome-associated coronavirus with dendritic cells. J. Gen. Virol. 87 (Pt 7), 1953-1960 (2006).

37. Yen, Y.T., Liao, F., Hsiao, C.H., Kao, C.L., Chen, Y.C. \& Wu-Hsieh, B.A. Modeling the early events of severe acute respiratory syndrome coronavirus infection in vitro. J. Virol. 80, 2684-2693 (2006). 